\title{
De l'observation du microclimat urbain à la modélisation intégrée de la ville
}

\author{
Grégoire Pigeon, Aude Lemonsu, Valéry Masson et Julia Hidalgo \\ Météo-France - Centre national de recherches météorologiques (CNRM) \\ 42, avenue Gaspard-Coriolis - 31057 Toulouse Cedex 1
}

\section{Résumé}

La manifestation la plus connue du microclimat généré par les villes, en particulier lors de canicule, est l'excès des températures, appelé îlot de chaleur urbain (il peut varier de $2^{\circ} \mathrm{C}$ pour une ville de 1000 habitants à $12{ }^{\circ} \mathrm{C}$ pour une ville de plusieurs millions d'habitants). Cet article passe en revue ce qui caractérise les modifications du climat en zones urbaines et explique les processus qui les gouvernent. D'après la littérature existante, principalement consacrée au climat urbain en période estivale, la modification du climat en ville ne résulte pas tant de la source additionnelle de chaleur dégagée par les activités humaines que du changement des propriétés de la surface - imperméabilité des revêtements, matériaux de grande capacité thermique et retrait de la végétation créant un environnement propice au piégeage du rayonnement solaire. es villes sont le lieu d'un micro-climat dont la caractéristique principale est l'îlot de chaleur urbain. Ses conséquences sur le confort des citadins sont connues depuis longtemps. En 1885, Guy de Maupassant décrit, dans Bel Ami, des Parisiens cherchant fréquemment la fraîcheur dans les parcs, afin d'échapper à la chaleur insoutenable de la ville ! Comparé à la violence d'un cyclone, d'une tempête ou d'un orage, le souci de confort urbain peut paraître un enjeu dérisoire. Cependant, ce microclimat a des conséquences sanitaires sur les populations, du fait notamment de l'augmentation du stress thermique que le corps doit affronter. Elles furent importantes lors de la canicule de 2003, et les victimes furent proportionnellement plus nombreuses en ville où les températures nocturnes peinaient à redescendre en dessous de $25^{\circ} \mathrm{C}$.

Les zones urbaines sont aussi un lieu d'émission de polluants. Modifiant l'écoulement atmosphérique, elles en affectent la dispersion. D'autres phénomènes météorologiques, comme la formation de brouillard, de pluie ou de neige, sont influencés par l'urbanisation des surfaces et les émissions associées. Enfin, elles sont des zones sensibles en cas d'accident industriel et des cibles potentielles d'attaques terroristes avec rejets toxiques dans l'atmosphère. Savoir prévoir les écoulements atmosphériques en milieu urbain devient une nécessité d'autant plus impérative qu'une proportion sans cesse croissante de la population et des activités socioéconomiques se trouve en ville.

Très prochainement, la résolution horizontale des modèles numériques de prévision du temps sera si fine que des mailles entièrement urbanisées en surface apparaîtront dans leurs grilles. Dans le cas du modèle Arome ${ }^{(1)}$ dont le maillage sera de $2,5 \mathrm{~km}$ de résolution, les grandes agglomérations françaises occuperont plusieurs points de grille. C'est dans cette perspective qu'a été développé le modèle Town Energy Balance [TEB] (Masson, 2000), dédié au calcul des échanges entre les surfaces urbanisées et l'atmosphère, et que deux campagnes de mesures ont été menées en France ces dernières années. Cellesci avaient pour objectif d'accroître notre connaissance du climat urbain et de réunir les observations nécessaires à l'évaluation des modèles numériques. La première campagne, CLU-Escompte ${ }^{(2)}$ (Mestayer et al., 2005), a eu lieu à Marseille en juin et juillet 2001 et a rassemblé plusieurs équipes françaises et internationales. Parmi les objectifs de CLU-Escompte, figuraient les études de la variabilité du bilan d'énergie en fonction des couverts urbains et de la variabilité spatio-temporelle de l'îlot de chaleur urbain. Plus récemment, une deuxième campagne, Capitoul $^{(3)}$, a eu lieu sur Toulouse pendant un cycle annuel complet (de février 2004 à mars 2005). Les attentes de ce programme concernaient le suivi du bilan d'énergie, de l'îlot de chaleur urbain, de la structure de la couche limite urbaine, et cela notamment en période hivernale, ainsi que les interactions entre la dynamique atmosphérique et les particules d'aérosols.

(1) Arome sera le prochain système numérique de prévision à aire limitée de Météo-France.

(2) CLU est le sigle pour Couche limite urbaine, et Escompte pour Expérience sur site pour contraindre les modèles de pollution atmosphérique et de transport d'émissions.

(3) Capitoul est le sigle pour Canopy and Aerosol Particles Interactions in Toulouse Urban Layer. 
-. L'article présente ensuite les résultats de deux campagnes françaises, CLU-Escompte et Capitoul. Pendant Capitoul, une comparaison, particulièrement originale, a pu être faite entre la consommation urbaine d'énergie et les observations des échanges entre la surface du centreville de Toulouse et les premières couches d'atmosphère. Elle a démontré le poids des dégagements d'énergie par l'activité humaine (chauffage surtout) sur le bilan d'énergie pendant la période hivernale. La comparaison des résultats avec ceux obtenus par le modèle TEB (Town Energy Balance) couplé à un modèle atmosphérique a révélé que TEB contribuait avec succès à la simulation du microclimat urbain. Cela a fait émerger l'idée d'une modélisation intégrée de la ville réunissant d'autres modèles, prenant chacun en compte une composante isolée du système urbain (microclimat, hydrologie, propagation du bruit, habitat).

\section{Abstract}

\section{From urban climate observation to} integrated modelling of the city

The best known aspect of urban microclimate is the "heat island" which can range from $2^{\circ} \mathrm{C}$ for a village of 1,000 inhabitants to $12^{\circ} \mathrm{C}$ for a large city. This article examines the changes caused by urbanisation and how they are brought about. Current literature, chiefly concerned with urban climate in summertime ascribes the changes, not so much to human activities as to changes of surface characteristics of retention of moisture and heat, and of albedo.

This article gives the results of two campaigns, CLU-Escompte and Capitoul. The latter tried to measure urban energy consumption and exchanges between the surface in the centre of Toulouse and the boundary layer. It showed clearly the overall heating caused by human energy use during wintertime. The results compared favourably with those from the Town Energy Balance atmospheric model. Thus it is now possible to visualise a model combining all the components of the urban system, microclimate, hydrology, noise propagation and buildings.
Cet article a pour but de dresser un bref état des connaissances sur le microclimat urbain en mettant notamment l'accent sur les résultats originaux obtenus à partir de ces deux campagnes récentes. Ainsi, on présentera d'abord les caractéristiques principales du microclimat urbain.

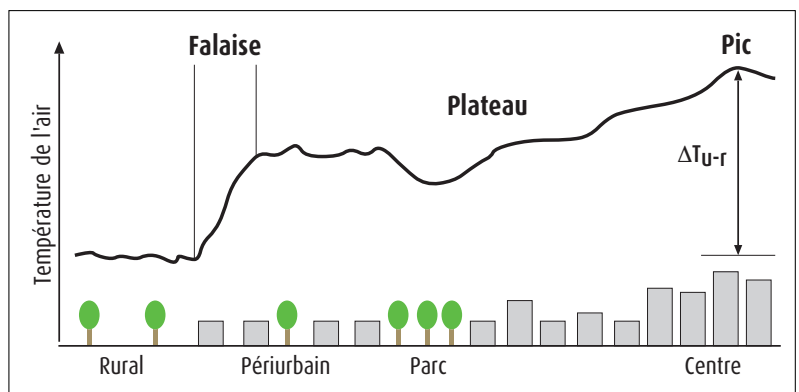

Figure 1 - Variation spatiale de la température de l'air le long d'une coupe transversale d'une zone urbanisée (adaptation de Oke, 1987). mis sur la présentation des processus qui conduisent à la mise en place de ce microclimat. Dans la continuité de ces deux premières parties, la représentation numérique de ces processus sera exposée. Enfin, quelques perspectives d'évolution de la recherche dans ce domaine seront esquissées.

\section{Le microclimat urbain}

Après une définition de l'îlot de chaleur urbain (ICU), principal élément du microclimat des villes, ce chapitre passe en revue ses principales manifestations : modifications des températures en surface du fait de l'urbanisation, stratification verticale en forme de dôme et écoulement perturbé par un régime de convergence généré par la ville.

\section{Températures en surface}

L'îlot de chaleur urbain est le principal élément du microclimat des villes. Il désigne l'excès des températures de l'air que l'on observe régulièrement, près du sol, dans les zones urbaines, en comparaison avec les zones rurales qui les entourent (figure 1).

Selon plusieurs études rassemblées par Oke (1987), les maxima d'intensité de l'ICU peuvent aller de $2{ }^{\circ} \mathrm{C}$ pour une ville de 1000 habitants à $12^{\circ} \mathrm{C}$ pour une ville de plusieurs millions d'habitants.

La notion d'ICU est intimement liée à une vision monocentrique de l'espace urbain, avec un centre-ville unique ainsi qu'à une vision urbanistique historique pour laquelle la limite entre la ville et son environnement naturel est très marquée. Ainsi, selon Oke (1987), la structure spatiale de l'ICU est concentrique autour du centre-ville et caractérisée par trois couronnes (figure 1). À l'extérieur, une couronne étroite correspondant à un intense gradient de température à la transition entre la ville et la campagne qu'il dénomme littéralement « falaise » (traduction de l'anglais cliff). Dans cette zone de transition, des gradients de $1^{\prime}$ ordre de 0,3 à $0,4{ }^{\circ} \mathrm{C}$ par 100 mètres ont été mesurés. La deuxième couronne dénommée «plateau » est la plus large. Elle est marquée par des gradients faibles et une tendance progressive au réchauffement au fur et à mesure que l'on s'approche du centre-ville. Enfin, le « pic » correspond à la zone du centre- ville. Bien sûr, l'écart de température entre le centre-ville et les zones rurales dépendra des caractéristiques architecturales de la ville (comme son étendue, la densité et la hauteur des bâtiments) et de l'espace rural pris comme référence. Enfin, l'espace urbain d'aujourd'hui est plus diffus et plus hétérogène. Les grandes villes ont phagocyté les noyaux villageois qui les entouraient et les limites de la zone urbaine sont floues. Il en résulte une structure d'îlot de chaleur souvent plus compliquée.

Figure 2 - Évolution de la température en deux points de Toulouse, le 25 février 2005 , pendant la campagne Capitoul.

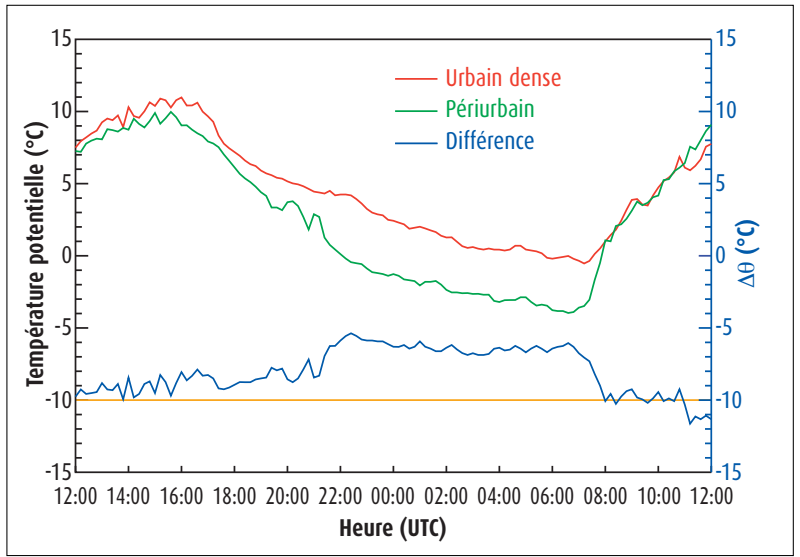


L'ICU a une variabilité journalière récurrente, son intensité est en général plus forte la nuit que le jour (figure 2). Il s'amplifie ou se forme progressivement lors de la période de refroidissement nocturne, en réponse à un taux de refroidissement plus faible dans les zones les plus urbanisées par rapport aux zones périphériques. Dans la majorité des cas, le maximum d'ICU semble se situer quelques heures après le coucher du soleil, puis l'ICU diminue rapidement après le lever du soleil. L'intensité de l'ICU diminue avec la vitesse du vent. On constate que l'ICU disparaît pour des vents supérieurs à $11 \mathrm{~m} . \mathrm{s}^{-1}$. En présence d'un vent modéré (3 à $6 \mathrm{~m} \cdot \mathrm{s}^{-1}$ ), le champ de température prend la forme d'un panache étiré selon l'axe du vent. En cas de vent très

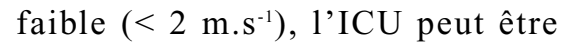
multicellulaire et sa forme varie selon le mode d'occupation des sols. L'intensité de l'ICU diminue lorsque la nébulosité augmente. Les nuages interviennent en modifiant le refroidissement radiatif nocturne pendant lequel se forme l'ICU. L'influence de la saison a été étudiée sur des villes du climat tempéré, mais aussi d'autres types de climat (Mexico, Le Caire, par exemple). Il est rapporté dans ces études que les ICU sont plus fréquents en été, qu'ils tendent à se former plus tard en hiver et à décroître moins vite en matinée. Enfin, les ICU sont plus faibles et moins fréquents en période pluvieuse.

\section{Stratification verticale}

On observe aussi une influence de l'urbanisation sur la structure verticale de l'atmosphère. En milieu de journée, la propriété la plus remarquable de la couche limite urbaine (CLU) est l'accroissement de son épaisseur en comparaison avec la couche limite rurale - parfois jusqu'à 250 mètres de plus. L'influence de la ville sur la couche limite est cependant plus marquée la nuit. On observe fréquemment que l'excès de température au-dessus de la zone urbaine diminue avec l'altitude et s'annule à une hauteur comprise entre 100 et 500 mètres au-dessus du sol. Au-dessus de cette couche, on observe un îlot froid (figure 3). Ce phénomène, baptisé « crossover », est associé à une CLU nocturne
Figure 3 - Profils de température potentielle observés pendant la campagne Capitoul e 25 février 2005 à 22 h 00 UTC.

convective de quelques centaines de mètres surmontée d'une inversion dite élevée alors qu'une inversion de surface est observée en zone rurale. Cette stratification particulière de l'atmosphère au-dessus des zones urbaines et de leurs environs conditionne la dispersion des polluants émis dans l'air. Un autre effet notable est l'impact sur les méthodes naturelles de ventilation. En effet, en période chaude, lorsque les habitants des étages les plus élevés des grandes tours d'immeuble cherchent à rafrâ̂chir leur appartement par ventilation naturelle, la stratification thermique et la hauteur de l'appartement par rapport à l'inversion influencent fortement l'efficacité de la ventilation. Ainsi, pendant la canicule de 2003, il a été observé en région parisienne que le risque de surchauffe nocturne augmentait pour les étages élevés des immeubles les plus hauts (Rousseau, 2005).

\section{Écoulement perturbé}

Par analogie avec les phénomènes de brises thermiques côtières, l'existence d'une convergence associée à l'ICU a été très vite conceptualisée (Oke, 1987). Ce type de circulation peut avoir un impact sur la répartition des polluants à l'échelle régionale (Lemonsu et Masson, 2002), et son observation fut l'un des objectifs de la campagne Capitoul. Une situation de convergence de basse couche, associée à une divergence proche du sommet de la couche limite, fut documentée au cours de la campagne, notamment à partir de

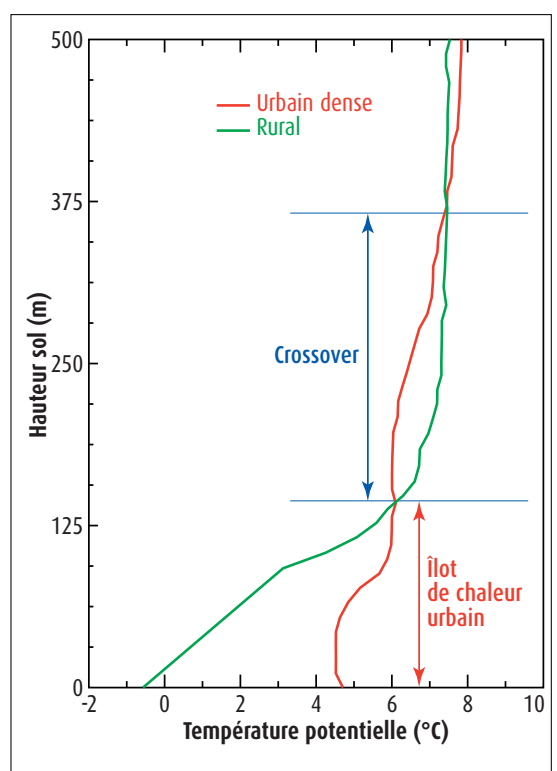

mesures aéroportées (figure 4), de radiosondage et de mesures par télédétection (Hidalgo et al., 2007).

\section{Processus à l'origine du microclimat urbain : le poids des échanges surface-atmosphère}

Quels sont les processus à l'origine du microclimat urbain? de jour? de nuit? Telles sont les questions abordées dans ce chapitre.

\section{Bilan d'énergie d'une zone urbaine}

L'îlot de chaleur urbain est un phénomène affectant les basses couches de l'atmosphère ; ainsi, les échanges d'énergie et de matières pour une surface urbaine sont des processus élémentaires à caractériser. L'équation de bilan d'énergie d'une
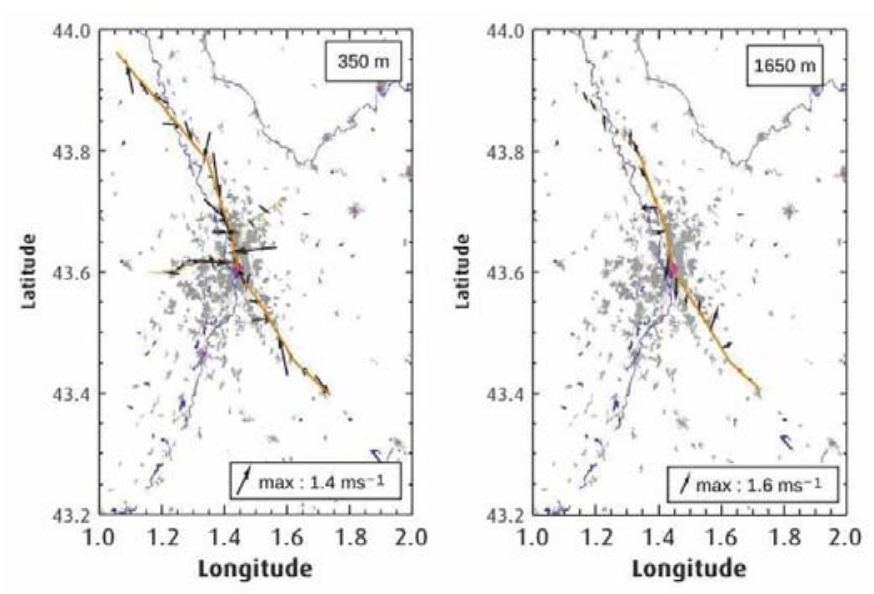

Figure 4 - Observations

de vent le long

de deux trajets avion

au-dessus de Toulouse

le 4 juillet 2004,

illustrant le phénomène

de brise urbaine.

À $350 \mathrm{~m}$ au-dessus du sol

(à gauche), on observe

une convergence

alors qu'une divergence

est observée à 1650 m (à droite). 
surface traduit l'équilibre entre les sources et les puits d'énergie. En ville, la surface est loin d'être plane et homogène et les éléments rugueux sont de grandes dimensions. Ainsi, au lieu de reprendre l'approche conventionnelle et d'établir le bilan d'énergie pour une surface fictive, Oke (1987) a adopté une approche plus macroscopique et a proposé d'établir le bilan d'énergie pour un volume de contrôle allant du sol au sommet des bâtiments. Le bilan d'énergie s'écrit alors selon l'équation suivante :

$\mathrm{Q}^{*}+\mathrm{Q}_{\mathrm{F}}=\mathrm{Q}_{\mathrm{H}}+\mathrm{Q}_{\mathrm{E}}+\Delta \mathrm{Q}_{\mathrm{S}}+\Delta \mathrm{Q}_{\mathrm{A}}$

où :

- Q* est le flux de rayonnement net, toutes longueurs d'onde confondues, reçu par la surface. Rigoureusement, il s'agit d'une densité de flux exprimée en W.m ${ }^{-2}$. C'est un terme compté positivement lorsque la surface gagne de l'énergie (le jour) et négativement lorsqu'elle en perd (la nuit). Il se décompose de la manière suivante : $\mathrm{Q}^{*}=\mathrm{K} \downarrow-\mathrm{K} \uparrow+\mathrm{L} \downarrow-\mathrm{L} \uparrow$ où $\mathrm{K} \uparrow$ et $\mathrm{K} \downarrow$ sont respectivement le flux solaire incident et réfléchi par la surface et $\mathrm{L} \downarrow$ et $\mathrm{L} \uparrow$ sont le flux infrarouge incident (émis par l'atmosphère) et remontant (émis par la surface).

- $\mathrm{Q}_{\mathrm{F}}$ est le dégagement de chaleur par l'ensemble des activités humaines, c'est un terme toujours positif.

- $Q_{H}$ et $Q_{E}$ sont respectivement les flux turbulents de chaleur sensible et latente. Ce sont généralement des termes puits et, par convention, ces termes sont comptés positivement lorsque le transfert d'énergie qu'ils représentent est dirigé de la surface vers l'atmosphère.

$-\Delta \mathrm{Q}_{\mathrm{s}}$ est la variation d'énergie interne par unité de temps et de surface de l'ensemble du volume de contrôle (incluant les bâtiments). En journée, c'est un terme puits (accumulation de chaleur par conduction) alors que la nuit il s'agit d'un terme source (relâchement de chaleur) qui compense les pertes radiatives. $-\Delta \mathrm{Q}_{\mathrm{A}}$ est l'advection moyenne de chaleur (l'advection moyenne d'humidité convertie en chaleur latente est incluse dans ce terme) à travers l'ensemble des parois du volume de contrôle.

Afin de faire ressortir la spécificité du bilan d'énergie d'une surface urbaine, nous décrirons l'évolution du bilan d'énergie sur un cycle journalier idéal en deux temps - le jour, puis la nuit - en le comparant avec celui d'une surface rurale fortement végétalisée (figure 5).

Figure 5 - Présentation du bilan d'énergie en zone rurale (à gauche) et en zone urbaine (à droite) le jour (en haut) et la nuit (en bas).
Naturellement, ces résultats sont à nuancer pour des villes plus atypiques comme, par exemple, Las Vegas, située au milieu d'un désert.

\section{Le jour}

Dans la journée, la raréfaction de la végétation et la faible disponibilité d'eau en surface, du fait de l'utilisation abondante de matériaux imperméables, conduisent à une forte réduction du flux de chaleur latente $Q_{E}$ en comparaison à la surface rurale (du fait du peu d'énergie perdue pour évaporer de l'eau en surface). Le rapport $\mathrm{Q}_{\mathrm{E}} / \mathrm{Q}^{*}$ est généralement compris entre 0,1 et 0,2 pour une zone urbaine dense, alors qu'il est généralement supérieur à 0,5 pour une zone rurale couverte de végétation. Ainsi, une plus grande quantité de l'énergie solaire est rendue disponible pour les autres termes puits du bilan d'énergie que sont l'accumulation de chaleur $\Delta \mathrm{Q}_{\mathrm{S}}$ (réchauffement des constructions par conduction) et le flux de chaleur sensible $Q_{\mathrm{H}}$ (réchauffement de l'atmosphère par flux turbulent). $\Delta \mathrm{Q}_{\mathrm{s}}$ est d'autant plus accentué que la géométrie tridimensionnelle de la surface urbaine (rue en forme de canyon) favorise le piégeage de rayonnement solaire par réflexion multiple et offre une plus grande surface d'échange. De plus, les propriétés thermiques des matériaux urbains favorisent l'accumulation de chaleur. En moyenne, le jour, $\Delta \mathrm{Q}_{\mathrm{S}}$ représente entre 30 et $60 \%$ de $Q^{*}$ pour une zone urbaine, soit en période estivale des valeurs entre 100 et 175 W.m. ${ }^{-2}$. De plus, le terme anthropique $\mathrm{Q}_{\mathrm{F}}$ constitue une source additionnelle de chaleur qui s'élève entre 5 et $150 \mathrm{~W} . \mathrm{m}^{-2}$ selon

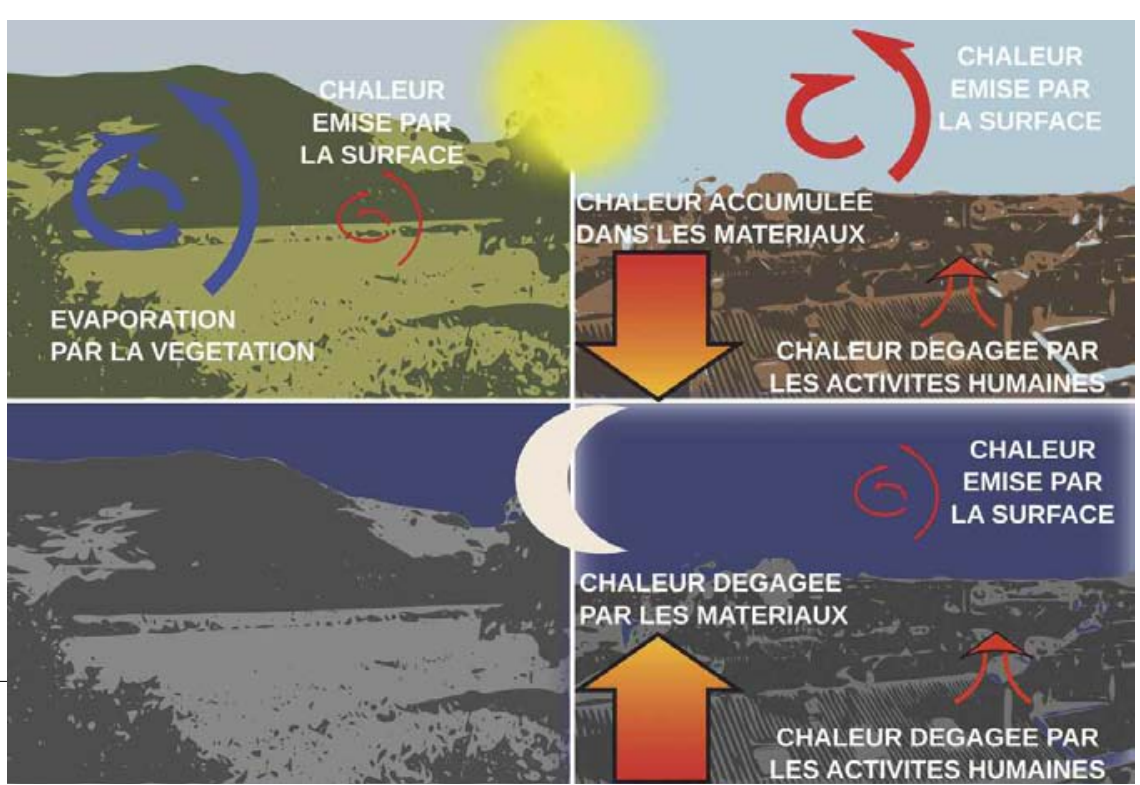

l'urbanisation et la saison. Ce dégagement de chaleur renforce le flux de chaleur sensible $\mathrm{Q}_{\mathrm{H}}$ qui représente généralement entre 35 et $60 \%$ de $Q^{*}$ selon l'urbanisation et la saison, soit entre 100 et 250 W.m ${ }^{-2}$.

De jour, l'urbanisation conduit donc principalement à une réduction de l'évaporation au profit de l'accumulation de chaleur dans les matériaux urbains.

\section{La nuit}

En soirée, alors que les échanges d'énergie sont très faibles en zone rurale, l'énergie accumulée dans la canopée urbaine ${ }^{(1)}$ est restituée vers l'atmosphère et maintient un chauffage convectif $\left(\mathrm{Q}_{\mathrm{H}}\right.$ positif jusqu'à $\left.100 \mathrm{~W} \cdot \mathrm{m}^{-2}\right)$ qui limite le refroidissement nocturne. C'est ainsi que se met en place l'ICU et que se maintient une faible couche limite convective au-dessus de la ville. De plus, ce phénomène est accentué par la géométrie encaissée des rues qui limite le refroidissement des parois (route et murs) par les pertes radiatives nettes (réduction du facteur de vue du ciel qui constitue un puissant puits radiatif).

En conclusion, la modification du climat dans les zones urbaines ne résulte pas tant de la source additionnelle de chaleur dégagée par les activités humaines que du changement des propriétés de la surface, qui conduit à une restitution lente de la chaleur accumulée le jour du fait des caractéristiques

(1) Par analogie aux forêts, le terme canopée met des bâtiments. urbaine désigne la couche entre le sol et le som- 
des matériaux urbains. Parmi les propriétés de surface, il faut retenir l'imperméabilisation des revêtements, le retrait de la végétation, la création d'une géométrie propice au piégeage du rayonnement solaire et l'utilisation en général de matériaux d'une grande capacité thermique.

\section{Campagne CLU-Escompte : le cas d'une ville côtière}

Jusqu'à présent, les variations spatiales et temporelles de l'ICU avaient été étudiées séparément au moyen de deux types de dispositifs expérimentaux. Respectivement, il s'agissait de véhicules instrumentés explorant la variabilité de température selon les diverses zones d'une agglomération ou d'un nombre restreint (deux ou trois) de stations situées en des zones plus ou moins urbanisées pour caractériser la variation temporelle de l'ICU. Constatant cette déficience, il a été décidé de déployer un réseau de vingt stations automatiques sur Marseille pendant la campagne CLUEscompte permettant une observation continue de l'ICU en différents points de la ville (Pigeon et al., 2006). Afin d'optimiser la disposition du réseau de stations,

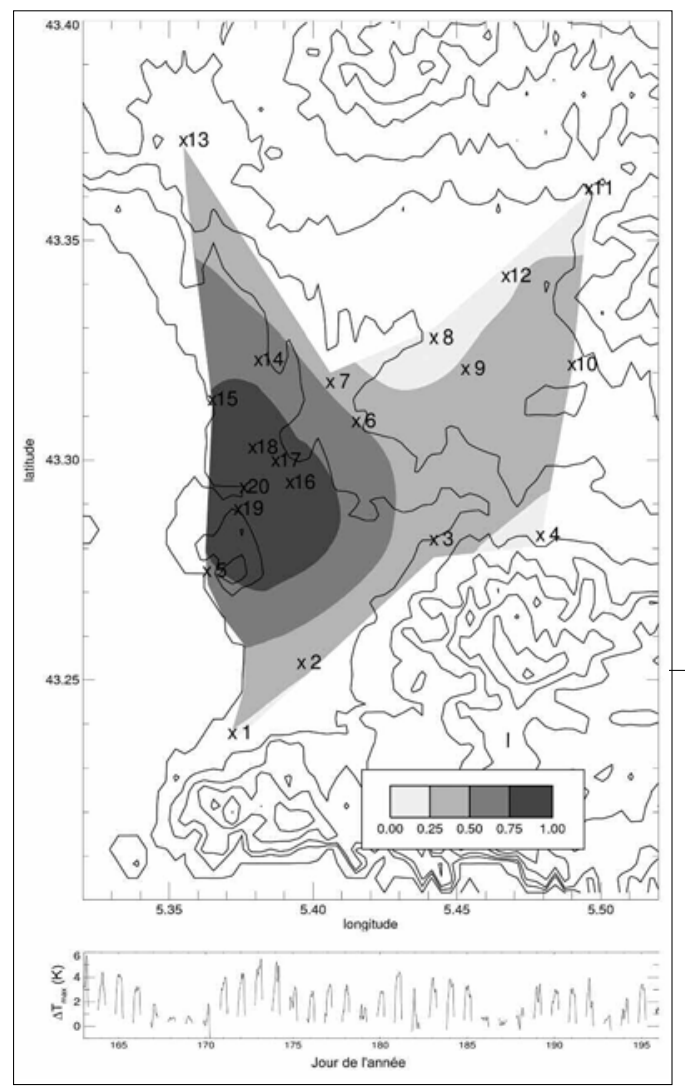

\section{Optimisation
de la disposition
du réseau de stations}

Afin d'optimiser la capacité du réseau de stations urbaines à capturer les caractéristiques spatio-temporelles de l'ICU sur Marseille, une simula-

tion numérique avec le modèle atmosphérique Méso-NH a été réalisée, en utilisant le schéma TEB (Masson, 2000) pour le calcul des échanges surface urbaine/ atmosphère. La simulation s'est faite sur quatre grilles emboîtées. Le plus petit domaine couvrait l'agglomération de Marseille à une résolution de 250 m. La simulation concernait trois jours consécutifs de la précampagne Escompte en juin et juillet 2000. Ce choix représentait un très bon échantillonnage des conditions météorologiques possibles dans la région à cette période de l'année : régime de mistral modéré, brise de mer

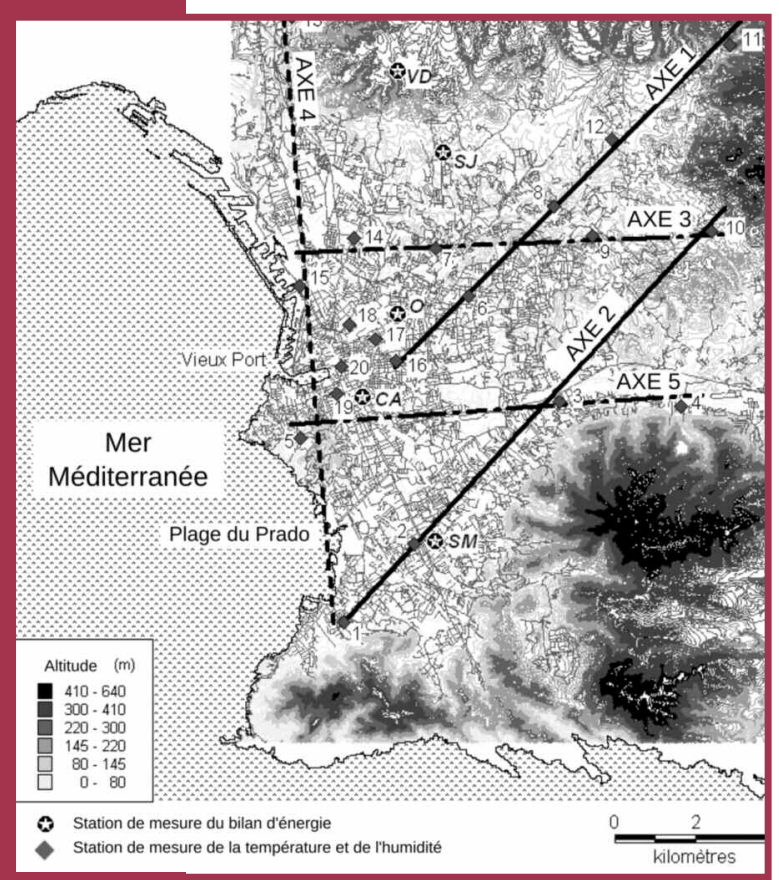
et régime de sud-est. De plus, la qualité de la simulation a pu être évaluée en la confrontant aux observations de la précampagne. Une attention toute particulière a été portée sur la capacité de la simulation à reproduire la variabilité spatiale de la température entre la zone urbaine et les zones environnantes. Ensuite, des informations synthé tiques et statistiquement pertinentes de la simulation sur la zone urbaine ont été extraites par une analyse en composante principale $(\mathrm{ACP})$. La disposition du réseau a été choisie dans le but d'échantillonner les structures spatiales et temporelles les plus significatives des champs de température et d'humidité (figure 6). À l'issue de la campagne de l'été 2001, les mesures de température et d'humidité des vingt stations du réseau ont également été analysées avec une ACP, mais de manière disjointe pour le jour et pour la nuit.

Figure 7 - Premier mode de variation

spatio-temporelle de la température nocturne pendant CLU-Escompte qui présente une structure d'îlot de chaleur urbain idéalisé. En haut, sa variation spatiale normalisée a une forme concentrique autour du centre-ville (station 20). L'échelle représente I'anomalie normalisée de température par rapport à une station de référence (ici la station 8). En bas, l'évolution temporelle associée au premier mode est représentée par l'anomalie de température de la station 20. Elle présente une périodicité journalière d'intensification de l'anomalie en début de nuit et de diminution en deuxième partie de nuit.
Figure 6 - Disposition du réseau d'observation de l'îlot de chaleur urbain sur Marseille pendant CLU-Escompte. Cinq axes de mesures ont été optimisés en fonction des résultats d'une simulation numérique de trois jours sur la ville de Marseille.

une méthode originale a été développée (voir l'encadré « Optimisation de la disposition du réseau de stations » cicontre). L'analyse en composante principale a ensuite été faite afin d'extraire des mesures les principaux modes de variation spatio-temporelle. La nuit, le premier mode est apparu sous la forme d'un ICU idéalisé dont la structure spatiale est concentrique autour du centre-ville de Marseille (figure 7). Son évolution temporelle prend la forme d'une série temporelle périodique qui présente en chaque début de nuit une intensification de l'écart de température entre le centreville et ses alentours à laquelle succède une période d'homogénéisation au cours de la deuxième partie de la nuit. Le jour, le phénomène d'ICU n'est pas observé et le champ de température, parallèle à la côte, est principale-ment dominé par les interactions mer/continent.

La structure spatiale de cet ICU idéalisé, mis en évidence pour la période nocturne, a ensuite été analysée en fonction de paramètres quantifiables de l'urbanisation. Autour de chaque site de mesure $(500 \mathrm{~m})$, la fraction couverte par les surfaces imperméables (bâtiments, routes, trottoirs, parkings) et le rapport entre la hauteur des bâtiments et la largeur des rues (appelé rapport d'aspect) ont été 
évalués à partir d'une base de données géographiques. Une très bonne corrélation a été démontrée entre la structure spatiale de l'ICU idéalisé et la fraction de surface couverte par des matériaux imperméables ainsi qu'avec le rapport d'aspect des rues entourant les sites de mesure (figure 8). Le jour, par contre, la corrélation entre la température et la fraction occupée par les surfaces imperméables était faible, et le critère le plus pertinent était la distance à la mer. Ces mêmes corrélations ont été retrouvées dans l'analyse de la simulation utilisée initialement pour optimiser la disposition du réseau dans laquelle des comportements très similaires ont été observés pour la température. Ce résultat souligne à la fois la validité de l'outil numérique utilisé, de la méthode mise en place pour optimiser l'implantation du réseau de stations et la stabilité dans le temps des relations entre les caractéristiques de la surface urbaine et la variabilité du champ de température puisque la simulation et les observations correspondaient à deux années différentes (2000 et 2001).

\section{Campagne Capitoul : bilan anthropique}

La campagne Capitoul, qui s'est déroulée à Toulouse et a duré une année entière, a permis d'étudier en détail l'îlot de chaleur urbain en période hivernale, jusqu'alors peu documenté. Les mesures ont mis en évidence l'importance du terme anthropique, qu'il est possible d'estimer de deux manières - à partir des mesures des termes du bilan d'énergie et par inventaire des consommations d'énergie - puis d'en étudier les variations.

\section{Estimation par mesures des termes du bilan d'énergie}

Jusqu'à très récemment, peu d'études s'étaient intéressées au bilan d'énergie des surfaces urbaines en hiver. Ce fut aussi l'un des objectifs de la campagne Capitoul durant laquelle le bilan d'énergie d'une année complète (février 2004 mars 2005) fut mesuré grâce à une station de mesures implantée en centreville de Toulouse sur une toiture-terrasse (figure 9). Les observations collectées ont été utilisées pour estimer le terme $\mathrm{Q}_{\mathrm{F}}$ de dégagement de chaleur par les activités humaines. En partant de l'équation du bilan d'énergie (équation 1) et en négligeant le terme d'advection $\Delta \mathrm{Q}_{\mathrm{A}}$ suite aux

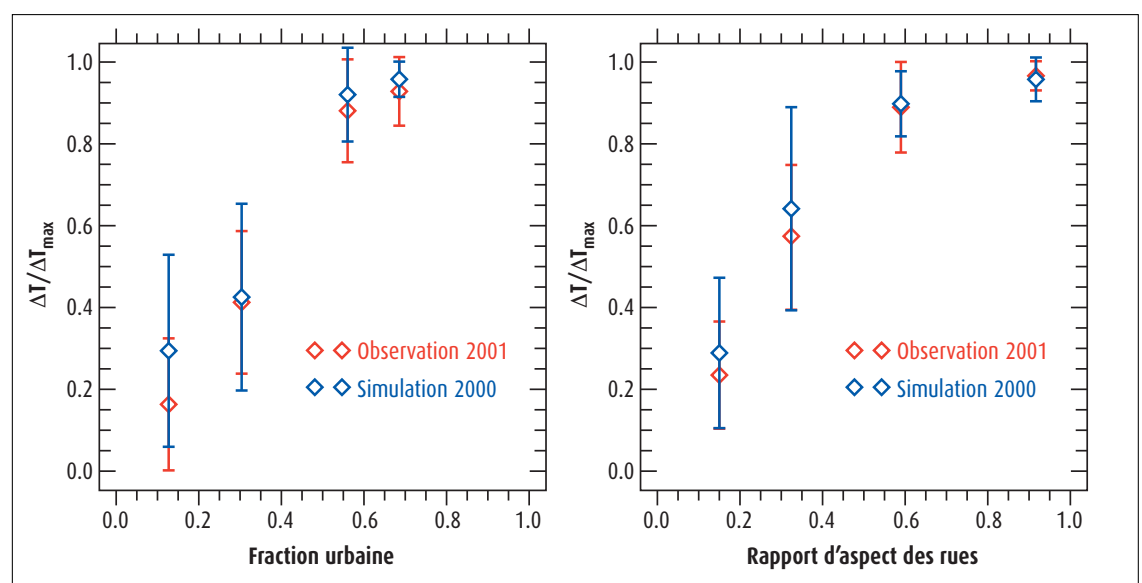

Figure 8 - Relation entre la variation spatiale du premier mode de variation spatio-temporelle de la température nocturne (représentée spatialement sur le graphique du haut de la figure 4) en fonction de la densité de bâti (à gauche) et de l'encaissement des rues (à droite). Sur ces graphiques, les comportements des observations de 2001 (noir) et de la simulation de 2000 (gris) sont représentés. Les valeurs selon l'axe des ordonnées évoluent des stations les plus froides aux plus chaudes.

résultats de Pigeon et al. (2007a) sur Marseille, il est établi que le résidu des mesures, $Q^{*}-\left(Q_{H}+Q_{E}\right)$ est égal à la différence entre le terme de stockage et le terme anthropique $\Delta \mathrm{Q}_{\mathrm{S}}-\mathrm{Q}_{\mathrm{F}}$. Ensuite, l'hypothèse classique a été faite que le relâchement de chaleur la nuit compense quasiment son accumulation pendant la journée. Ainsi, $\Delta \mathrm{Q}_{\mathrm{s}}$ intégré sur un ou plusieurs cycles journaliers tend à devenir un terme négligeable et le résidu intégré sur les mêmes périodes est ainsi égal à l'opposé du terme $\mathrm{Q}_{\mathrm{E}}$ de dégagement de chaleur des activités humaines. D'après des travaux antérieurs (Offerle et al., 2005), une précision de $\pm 10 \mathrm{~W} \cdot \mathrm{m}^{-2}$ peut être attendue sur ce terme par cette méthode d'estimation.

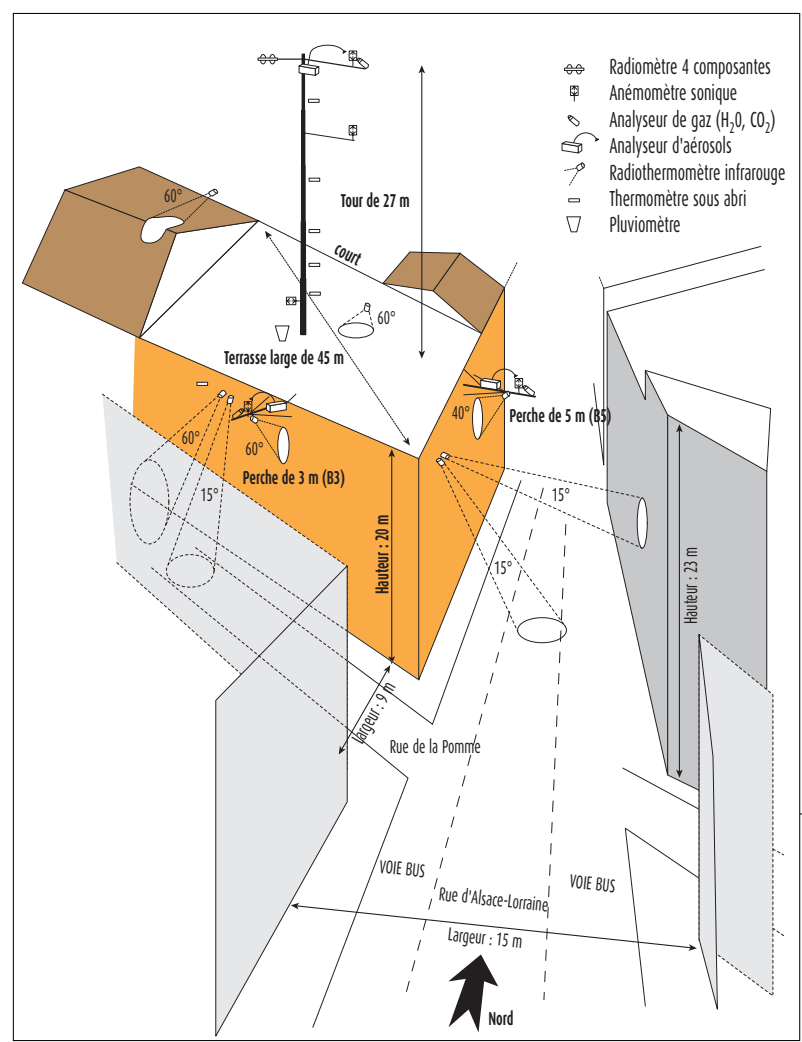

\section{Estimation par reconstitution des consommations d'énergie}

Indépendamment de la méthode précédente, un inventaire de la consommation d'énergie a été constitué sur l'agglomération toulousaine, pour la période de la campagne. Il repose sur des données de consommation réelle d'électricité (pas de temps horaire) et de gaz (pas de temps journalier) qui représentent $90 \%$ des consommations d'énergie liées au secteur résidentiel dans l'agglomération. Pour les autres sources d'énergie fossile (fioul, bois), des données annuelles (2001) de consommation ont été fournies par l'Observatoire régional de l'énergie en M i d i - P y réné es (Oremip). Pour ces sources d'énergie dont le mode d'utilisation est proche de celui du gaz (principalement chauffage et éventuellement production d'eau chaude sanitaire), le cycle annuel normalisé de la fourniture de gaz a été appliqué afin de préciser dans le temps leur consommation. La localisation à une échelle spatiale plus fine des consommations a été réalisée en

Figure 9 - Schéma de l'instrumentation du site central pendant la campagne Capitoul. Le bilan d'énergie était mesuré au sommet de la tour de 27 mètres. 
deux étapes. Premièrement, les données disponibles pour l'ensemble de l'agglomération ont été réparties selon des estimations statistiques disponibles auprès de l'INSEE à une échelle plus fine (typiquement un quartier). À l'intérieur de ce maillage irrégulier, les consommations ont été localisées plus finement sur une grille régulière de 100 mètres de résolution en fonction de la densité de bâtiments. La consommation d'énergie liée au trafic routier a également été prise en compte. Une base de données géographiques, disponible auprès de la mairie, concentre les trafics journaliers en moyenne annuelle pour chacune des sections de rue de la commune ainsi que pour les grands axes. C'est à partir de cette information et de l'estimation de la consommation de carburant obtenue auprès de l'Institut national de recherche sur les transports et leur sécurité (Inrets) qu'a été calculé le dégagement de chaleur par le trafic sur une grille de 100 mètres. Au final, des cartes de dégagement de chaleur ont été établies, comme par exemple la figure 10 qui montre le dégagement de chaleur moyen pendant la période hivernale.

\section{Variation du terme anthropique sur Toulouse}

Les estimations réalisées à partir des deux méthodes (voir les deux sections précédentes) ont été comparées sur une zone géographique de 500 mètres de rayon autour du site de mesure (figure 11). Pendant la période hivernale, $\mathrm{Q}_{\mathrm{F}}$ atteint des valeurs de l'ordre de $60 \mathrm{~W} \cdot \mathrm{m}^{-2}$ pour cette zone du centre-ville, et les estimations réalisées à partir des mesures se comparent très bien à l'inventaire de consommation d'énergie. Pendant la période estivale, l'estimation à partir des observations a tendance à sous-estimer les valeurs de $\mathrm{Q}_{\mathrm{F}}$ calculées à partir de l'inventaire (de l'ordre de $20 \mathrm{~W} \cdot \mathrm{m}^{-2}$ ). C'est aussi pendant cette période que l'on peut s'attendre à de moins bons résultats à cause de :

- la diminution logique de $\mathrm{Q}_{\mathrm{F}}$ en été ;

- la sous-estimation chronique des flux turbulents par les méthodes de mesure.

Cette comparaison d'un inventaire de la consommation d'énergie avec des mesures du bilan d'énergie sur une même période et un même site confère à cette étude son caractère très original. Pour le centre-ville de Toulouse, $\mathrm{Q}_{\mathrm{F}}$ varie entre 100 et $25 \mathrm{~W} \cdot \mathrm{m}^{-2}$ entre l'hiver et l'été, alors qu'il varie entre 25 et $5 \mathrm{~W} . \mathrm{m}^{-2}$ dans les zones périurbaines pour les mêmes périodes.
Figure 10 - Dégagement de chaleur par les activités humaines sur Toulouse pendant l'hiver 2004-2005 à une résolution de 100 mètres.

\section{Processus urbains dans les modèles d'écoulement atmosphérique}

L'acquisition d'une meilleure connaissance des échanges surfaces urbaines-atmosphère contribue directement aux améliorations des modèles numériques développés pour la prévision du temps et des caractéristiques environnementales des zones urbaines. C'est dans cette perspective qu'a été développé le modèle Town Energy Budget [TEB], (Masson, 2000).

Ce modèle ne traite que des surfaces spécifiques du milieu urbain que sont les routes (ou parkings) et les bâtiments. Les surfaces végétales qui sont aussi présentes en ville sont à traiter par un autre modèle tel que Isba (Noilhan et Mahfouf, 1996). Dans TEB, l'unité de base est la rue canyon : il s'agit d'une rue entourée de deux murs et d'un toit (figure 12). Cette rue est considérée d'une longueur

Figure 11 - Comparaison entre les estimations du dégagement de chaleur par les activités humaines en centre-ville de Toulouse.

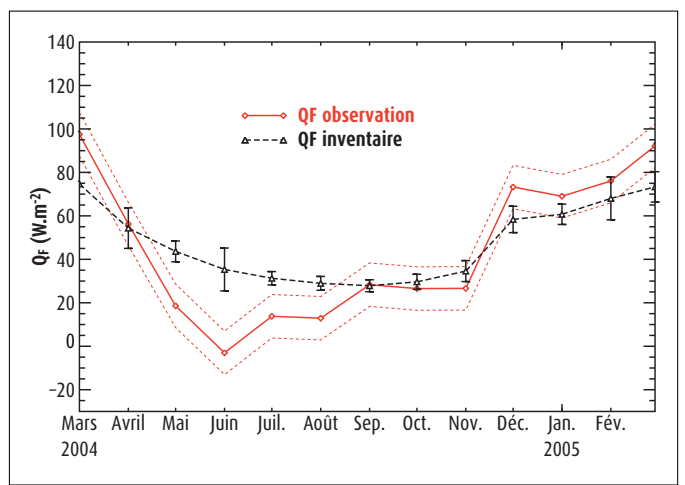

Figure 12 - Représentation schématique du modèle TEB (Masson, 2000).

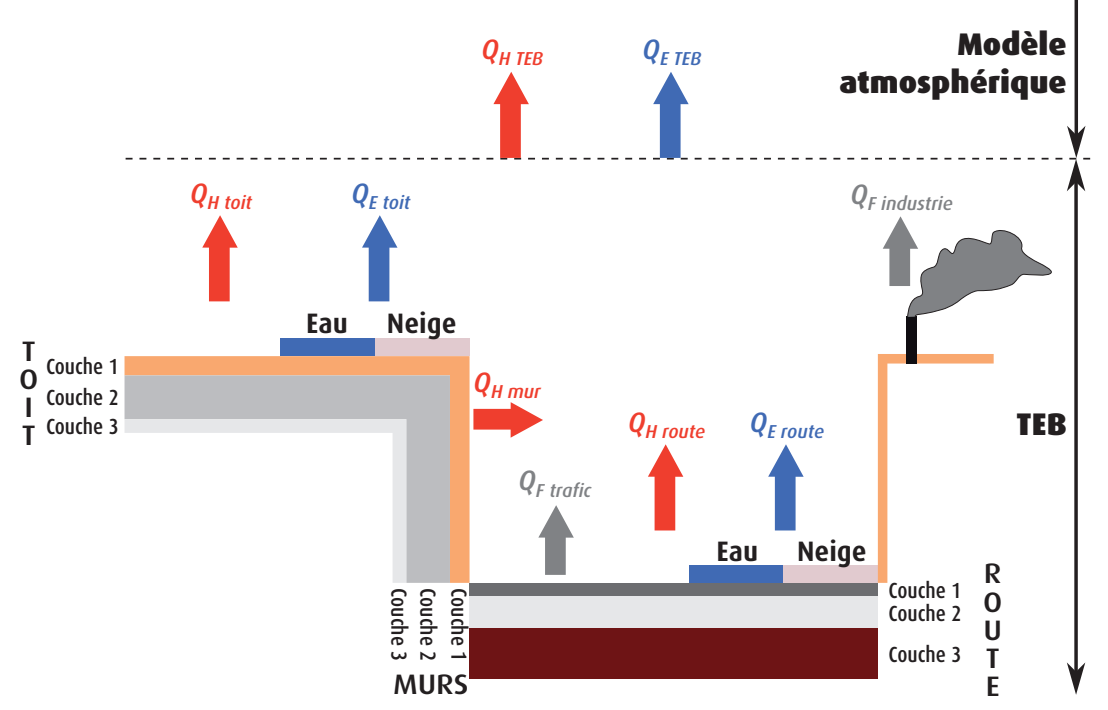


infinie et les intersections sont négligées. Pour tenir compte des effets de la géométrie sur le rayonnement, toutes les directions de rue sont considérées équiprobables (même en situation de ville réelle) et la largeur des rues ainsi que la hauteur des bâtiments sont ajustables. Pour chacune des surfaces élémentaires (route, mur, toit), un bilan d'énergie est calculé. Le transfert de chaleur par conduction dans ces structures est également établi. Les dégagements de chaleur par le trafic et les activités industrielles sont prescrits. Dans le cas de simulations sur des villes réelles, il est nécessaire d'alimenter le modèle par des couches d'informations localisées géographiquement qui précisent les caractéristiques de la morphologie urbaine, les propriétés thermiques des matériaux de construction ainsi que le dégagement de chaleur par les activités humaines (autres que le chauffage). Actuellement, ce travail est réalisé au cas par cas selon les besoins de recherche. Enfin, les dégagements de chaleur associés au chauffage des bâtiments sont représentés implicitement en fixant un seuil de température minimal à l'intérieur du bâtiment. En sortie du modèle TEB, on peut alimenter un modèle atmosphérique par les flux de chaleur, de vapeur d'eau et de quantité de mouvement (figure 12). La température et l'humidité dans les rues sont également calculées pour appréhender le microclimat urbain.

Un premier exercice d'évaluation du modèle a été mené avec des jeux de données provenant de Vancouver et Mexico (Masson et al., 2002). Les deux campagnes CLU-Escompte et Capitoul ont permis l'acquisition d'autres observations très pertinentes pour l'évaluation du modèle sur des centres-ville européens. Ainsi, une étude qui a été menée sur Marseille (Lemonsu et al., 2004) a permis de faire progresser le modèle sur la représentation des échanges entre la rue et l'atmosphère audessus de la canopée urbaine. À l'issue de Capitoul, les données de bilan d'énergie et l'inventaire de consommation d'énergie ont été exploités, pour les saisons d'automne et d'hiver, afin d'évaluer la capacité du modèle TEB à prévoir le terme de chauffage à l'intérieur des bâtiments (Pigeon et al., 2008). Un très bon comportement du modèle $\mathrm{a}$ été observé (figure 13) pour la reproduction de l'ordre de grandeur de ce terme tout comme celui de sa variation saisonnière. Des améliorations sont envisagées pour tenir compte des variations temporelles qui relèvent de l'activité économique et sociale (cycles

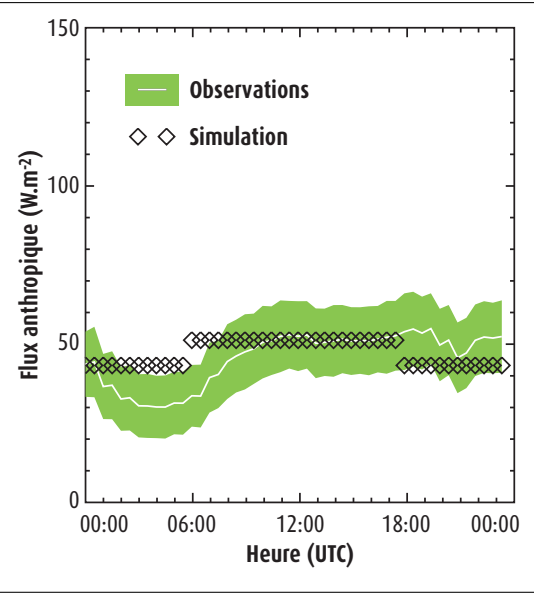

Figure 13 - Comparaison des observations du dégagement de chaleur par les activités humaines pendant l'automne 2004, en centre-ville de Toulouse, avec les résultats d'une simulation avec TEB.

diurne ou hebdomadaire). Une évaluation est également en cours sur des épisodes neigeux documentées lors de la campagne Muse, qui a eu lieu à Montréal à la fin de l'hiver 2005 (Lemonsu et al., 2008). Elle présente des résultats très encourageants pour la représentation de la neige en milieu urbain et son impact sur le bilan d'énergie dans le modèle TEB. Enfin, pour compléter cet effort d'évaluation déjà important, deux exercices d'intercomparaison de modèles sont en cours. Le premier se concentre sur la représentation des flux radiatifs dans l'infrarouge en collaboration avec l'Onera, l'École centrale de Nantes, l'Inra et le Centre d'études spatiales de la biosphère (Cesbio). Le deuxième est un effort international de comparaison des modèles dédiés aux surfaces urbaines piloté par le King's College de Londres.

L'utilisation du modèle TEB couplé à un modèle atmosphérique se révèle être à même de simuler le microclimat urbain. Par exemple, l'ICU a été correctement reproduit lors de simulations conduites sur Paris (Lemonsu et Masson, 2002). De même, en utilisant ce couplage, la sensibilité de l'écoulement atmosphérique régional à l'urbanisation de la surface a pu être montrée (figure 14). Ce type de couplage permet aussi de démonter les multiples interactions qui existent entre la dynamique de petite échelle et les épisodes de pollutions (Sarrat et al., 2006). Plus récemment, la représentation de la brise urbaine, bien que de faible ampleur, a été possible avec le modèle atmosphérique de recherche Méso-NH (Hidalgo et al., 2008) et la confrontation des résultats avec les observations collectées pendant Capitoul a confirmé le réalisme de cette simulation.

\section{Vers une modélisation intégrée de la ville}

Le climat urbain est une conséquence involontaire de l'action de l'homme. Audelà de cette prise de conscience, il est intéressant de dresser une perspective qui permettra d'en tenir compte dans la démarche d'urbanisation actuelle. L'adaptation de l'espace urbain à notre environnement changeant, comme le climat, est une préoccupation qui transparaît auprès des collectivités territoriales et de l'ensemble de la société, comme l'a montré la prospective Agora 2020 de la Drast $^{(1)}$. C'est dans ce contexte qu'a émergé l'idée d'une modélisation intégrée de la ville. Il s'agit de coupler des outils numériques spécifiques à la modélisation de composantes isolées du système urbain (microclimat, hydrologie, propagation du bruit, habitat) et de son environnement proche. Une plate-forme, qui simulera numériquement les principaux processus physiques intervenant dans le fonctionnement du système ville,

(1) Drast : Direction de la recherche et des affaires scientifiques et techniques.

Figure 14 - Champ de vent simulé avec Méso-NH sur l'agglomération parisienne. À gauche, on observe une convergence au niveau 200 m, alors qu'à droite on observe une divergence au niveau 2000 m. Par analogie au phénomène de brise marine, celui-ci est appelé brise urbaine.
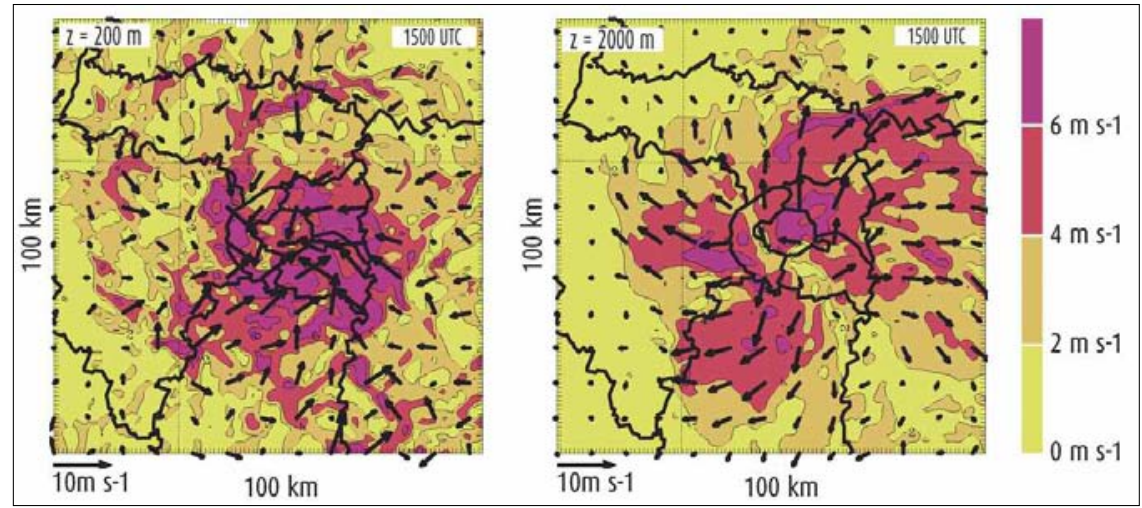
permettra de comprendre, quantifier et prévoir certaines de ses propriétés, en particulier ses bilans énergétiques et hydrologiques et ses conditions environnementales (notamment climat et qualité de l'air, mais également qualité des eaux, des sols et nuisances sonores). Le bilan énergétique prendra en compte notamment les échanges d'énergie entre les différents systèmes anthropiques et l'atmosphère, qui sont en interaction étroite. Par exemple, une part importante de la consommation énergétique dépend des conditions météorologiques (chauffage et climatisation, mais aussi transports, éclairage et différents usages domestiques et activités industrielles). La plate-forme visera également à évaluer le bilan carbone de l'agglomération et sa dépendance aux conditions climatiques actuelles et futures. Par une approche essentiellement fondée sur la physique et à l'aide d'une interface utilisateur appropriée, il sera possible d'évaluer l'impact sur le bilan énergétique et sur les conditions environnementales de différents facteurs comme les évolutions à moyen et à long termes des aménagements, du bâti, des transports, du changement climatique, ainsi que les impacts d'événements catastrophiques, qu'ils soient naturels, accidentels, ou des actes de malveillance et de terrorisme. Cela permettra, entre autres, d'identifier et d'évaluer de façon quantitative les mesures d'adaptation
Figure 15 - Représentation schématique d'une approche de modélisation intégrée de la ville.

pour atténuer les impacts du changement climatique et celles pour optimiser la consommation d'énergie et le bilan carbone d'un système urbain.

Les différents modules de la plate-forme (figure 15) devront permettre :

- de définir les scénarios de simulation : scénarios internes pour l'évolution propre au système ville (politiques publiques, projets d'aménagements), scénarios externes pour l'évolution des paramètres environnementaux au système (climat) ;

- d'activer les modèles qui traduisent ces scénarios en bases de données de conditions initiales et de conditions aux limites pour la simulation du système ville ;

- d'activer une simulation dynamique de l'évolution du système ville ;

- de synthétiser les résultats sous la forme d'indicateurs pertinents traduisant les impacts, la vulnérabilité ou l'adaptation du système ville.

\section{Bibliographie}

\footnotetext{
Hidalgo J., G. Pigeon et V. Masson, 2007: Urban breeze circulation during the Capitoul experiment: Experimental data analysis approach. Meteorology and Atmospheric - Physics, accepté pour publication.

- Lemonsu A. et V. Masson, 2002: Simulation of a summer urban breeze over Paris. Bound.-Layer Meteor., 104, 463-490.

- Lemonsu A., C. S. B. Grimmond et V. Masson, 2004: Modeling the surface energy balance of the core of an old mediterranean city: Marseille. J. Appl. Meteor., 43, 312-327.

- Lemonsu A. et al., 2005: Overview and first results of the Montreal Urban Snow experiment 2005. J. Appl. Meteor. and Climatol., 47, 59-75.

- Masson V., C. S. B. Grimmond et T. R. Oke, 2002: Evaluation of the Town Energy Balance (TEB) scheme with direct measurements from dry districts in two cities. - J. Appl. Meteor., 41, 1011-1026.

- Masson V., 2000: A physically-based scheme for the urban energy budget in atmospheric models. Bound.-Layer Meteor., 94, 357-397.

- Mestayer P. G., P. Durand et al., 2005: The Urban Boundary-Layer Field Campaign in Marseille (UBL/CLU-Escompte): Set-Up and first results. Bound.-Layer Meteor., 114, 315-365.

- Noilhan J. et J. F. Mahfouf, 1996: The ISBA land surface parameterisation scheme. Global and Planetary Change, 13, 145-159.

Offerle B., C. S. B. Grimmond et K. Fortuniak, 2005: Heat storage and anthropogenic heat flux in relation to the energy balance of a central European city centre. Int. - J. Climatol., 25, 1405-1419.

- Oke T. R., 1987 : Boundary Layer Climates, Methuen, London and New York, 435 p.

- Pigeon G., A. Lemonsu, N. Long, J. Barrié, P. Durand et V. Masson, 2006: Urban thermodynamic island in a coastal city analyzed from an optimized surface network. Bound.-Layer Meteor., 120, 315-351.

Pigeon G., A. Lemonsu, C. S. B. Grimmond, P. Durand, 0. Thouron et V. Masson, 2007: Divergence of turbulent fluxes in the surface layer: case of coastal city. Bound.-Layer Meteor., 124, 269-290.

Pigeon G., D. Legain, P. Durand et V. Masson, 2007: Anthropogenic heat releases in an old European agglomeration (Toulouse, France). Int. - J. Climatol.,, 27, 1969-1981.

- Rousseau D., 2005: Analyse fine des surmortalités pendant la canicule de 2003. La Météorologie, 8e série, 51, 16-22.

- Sarrat C., A. Lemonsu, V. Masson et D. Guedalia, 2006: Impact of urban heat island on regional atmospheric pollution. Atmos. Environ., 40, $1743-1758$.
} 